\title{
VELOS - A VR environment for ship applications: current status and planned extensions
}

\author{
A.I. Ginnis ${ }^{1}$, K.V. Kostas ${ }^{2}$, C.G. Politis ${ }^{2}$, and P.D. Kaklis ${ }^{3}$ \\ 1 School of Naval Architecture \& Marine Engineering (NAME) \\ National Technical University of Athens (NTUA) \\ 2 Dept. of Naval Architecture (NA), \\ Technological Educational Institute of Athens (TEI-A) \\ 3 Department of Naval Architecture, Ocean and Marine Engineering (NAME) \\ University of Strathclyde
}

\begin{abstract}
Virtual Environment for Life On Ships (VELOS) is a multiuser Virtual Reality (VR) system that supports designers to assess (early in the design process) passenger and crew activities on a ship for both normal and hectic conditions of operations and to improve the ship design accordingly [1]. Realistic simulations of behavioral aspects of crowd in emergency conditions require modeling of panic aspects and social conventions of inter-relations. The present paper provides a description of the enhanced crowd modeling approach employed in VELOS for the performance of ship evacuation assessment and analysis based on the guidelines provided by IMO's Circular MSC 1238/2007 [2].
\end{abstract}

\section{Introduction}

Under the impact of a series of events involving large number of fatalities on passenger ships [3], the International Maritime Organization (IMO) has developed regulations for new and existing passenger ships, including ro-ro passenger ships, requiring escape routes to be evaluated by an evacuation analysis described in IMO's Circular MSC 1238/2007, entitled Guidelines for evacuation analysis for new and existing passenger ships [2]. It is worth mentioning that, although the evacuation scenarios in [2] address issues related to the layout of the ship and passenger demographics, they do not address issues arising in real emergency conditions, such as unavailability of escape arrangements (due to flooding or fire), crew assistance in the evacuation process, family-group behavior, ship motions, etc. To heal such deficiencies, [2] adopts the mechanism of safety factors.

Much effort has been devoted to the development of sophisticated models for performing advanced evacuation analysis of passenger ships. As a result, around twenty such models and tools are available as reported in [4], [5]. A not-necessarily complete list should include the following tools:

1. AENEAS [6], a fast-performing simulation tool, allowing for large passenger populations. 
2. Maritime-EXODUS [7], a customization of the evacuation platform EXODUS that makes use of proprietary trial data for the behavior of passengers under conditions of list and heel.

3. IMEX [8], a ship evacuation model combining dynamics and human behavior model.

4. Evi [9], [10], a multi-agent evacuation simulation software package, utilizing the mesoscopic approach.

5. EVAC [11], a mustering simulation program that adopts the microscopic approach and utilizes data and knowledge stemming from EU-funded projects.

6. BYPASS [12], a simple cellular-automaton based model.

Crowd simulation is a complex task with issues related to collision avoidance, considering a large number of individuals, path planning, trajectories and so forth. Depending on the application, other requirements such as real-time simulation is needed to populate virtual environments in VR systems. Moreover, in order to provide a tool to simulate behavioral aspects of crowd in emergency conditions, panic aspects and social conventions of inter-relations are needed, $[13,14]$. In general, three approaches are used to model crowd motion. The Fluid model, where fluid equations, such as Navier Stokes equations, are used to model crowd flow [15-17]. The Cellular Automata (CA) model, which are discrete dynamic systems whose behavior is characterized by local interactions. Each CA is made up of a regular lattice of cells and at each unit of time the state of each cell is recalculated by the application of a set of rules to neighboring cells [18, 19]. The majority of crowd simulations employ the Particulate approach, which is also called the atomic approach. This is also the approach for crowd modeling used in VELOS and it is briefly presented in $\S 2.1$. The first pioneer work on this area was that of Reynolds [20] who worked on simulations of flocks of birds, herds of land animals and schools of fish. A later work of the same author [21] extends these concepts to the general idea of autonomous characters with an emphasis on animation and games applications. A Social force model for crowd simulation was introduced by Helbing and Molnár in [22]. They suggest that the motion of pedestrians can be described as if they are subject to social forces Acceleration, Repulsion and Attraction- which measure the internal motivation of individuals to perform certain actions. By combining these three forces they produce an equation for pedestrian's total motivation and finally the social force model. In [14] the social force model was applied to the simulation of building escape panic, with satisfactory results.

The paper is structured as follows: Section 2 presents VELOS's base: VRsystem, along with its major components and functionalities including a brief description of the employed crowd modeling approach for the performance of ship evacuation assessment \& analysis, while $\S 3$ is devoted to our proposed additions in steering behaviors and crowd modeling allowing their usage in ship evacuation analysis. Section 4 includes the presentation of ship evacuation test cases investigating the effects of crew assistance, passenger grouping and fire incidents. Furthermore, an additional test case demonstrating the effects of ship motions 
on passengers movement is also included. The last section is devoted to our ongoing work extending grouping behavior with dynamic characteristics.

\section{The VELOS system}

VELOS is based on VRsystem [1], a generic multi-user virtual environment, that consists of mainly two modules, the server and client modules connected through a network layer. Figure 1 provides a schematic overview of the VRsystem architecture. As depicted in this figure, users' participation in the virtual environment is carried out through the CLIENT module in the form of AVATARS enabling them to be immersed in the virtual world and actively participate in the evacuation process by interacting with agents and other avatars. On the other hand, system administrator utilizes the SERVER module for creating the virtual environment, setting all properties and rules for the scenario under consideration, e.g., scheduling of fire/flooding events, and awaits participants to connect to the system. Administrator's interaction may also take place during simulation phase.

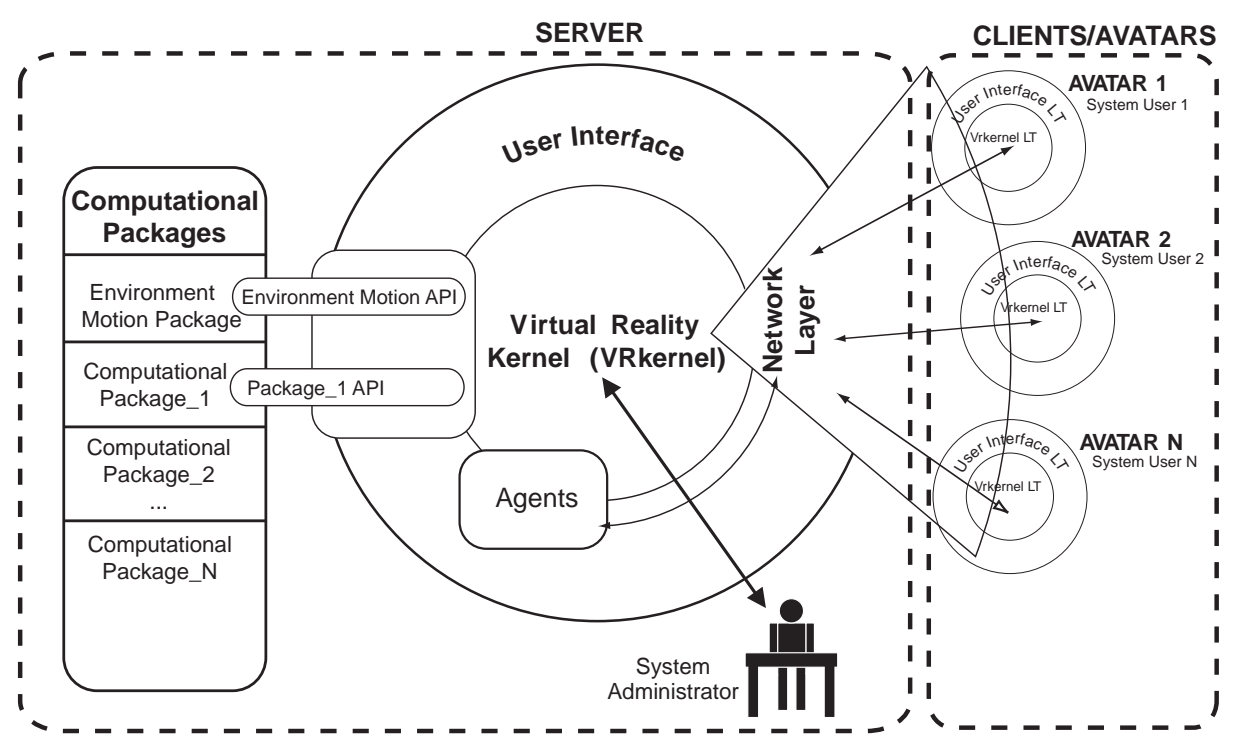

Fig. 1. The VRsystem Architecture

The server module comprises two major components, namely the VRkernel and the User-Interface, while the client module has a similar structure and comprises customized versions of them, referred to as VRkernelLT and UserInterfaceLT; see again Fig. 1. VRkernel is the core component of VRsystem platform in the server module. It can be thought of as a library of objects and 
functions suitable for materializing the synthetic world with respect to geometric representations, collision detection, crowd modeling, motion control and simulation, event handling and all other tasks related to visualization and scene organization. The core functionalities of VRkernel are provided by Open Inventor, an OpenGL based library of objects and methods used to create interactive $3 \mathrm{D}$ graphics applications.

\subsection{Crowd modeling for ship evacuation}

Crowd Modeling is a major part of VRkernel and, in view of VELOS areas of interest (evacuation, ergonomics, comfortability), it could be considered as the most significant of its components. It is based on agents, avatars, scene objects (such as obstacles) and steering behaviors technology. The term agent in VRkernel is used to describe autonomous characters, which "...combine aspects of an autonomous robot with some skills of a human actor in improvisational theater"; see [21]. Avatars are the system users' incarnation within the virtual environment and their major difference from agents is their controlling entity: humans for avatars vs. computer for agents. Avatars may take any role in the simulation; however they are more commonly used for controlling crew members in evacuation scenarios presented in the subsequent sections. Steering behaviors technology is the core of VRkernel's crowd modeling and is presented in the following paragraphs while enhanced crowd modeling features for ship evacuation are presented in section 3 .

The motion behavior of an agent is better understood by splitting it into three separate levels, namely action selection, steering and locomotion. In the first level, goals are set and plans are devised for the action materialization. The steering level determines the actual movement path, while locomotion provides the articulation and animation details.

Agents' autonomy is materialized within the steering level, where the steering behaviors technology is applied. Specifically, agents' autonomy is powered by an artificial intelligence structure, referred to in the pertinent literature as mind; see, e.g., [21,23]. The mind utilizes a collection of simple kinematic behaviors, called steering behaviors, to ultimately compose agent's motion. Specifically, for each time frame, the agent's velocity vector is computed by adding the previous velocity vector to the mind-calculated steering vector. This vector is a combination of the individual steering vectors provided by each associated steering behavior in agent's mind. For example, in Seek behavior the steering vector can be calculated as $\mathbf{f}=w \frac{\mathbf{q}-\mathbf{p}}{\|\mathbf{q}-\mathbf{p}\|}$, where $\mathbf{p}$ is agent's position, $\mathbf{q}$ is the seek point and $w$ is an appropriate weighting factor.

Nearly twenty steering behaviors have been so far implemented within VRkernel. These behaviors, based on the works by C.W. Reynolds [21] and R. Green [23], include: Seek, Arrive, Wander, Separation, Cohere, Leader Follow, Obstacle Avoidance 8$\}$ Containment, Path-following, Pursuit, Flee, Evade, offset\{Seek, Flee, Pursuit, Evade, Arrive $\}$; see also [24,25].

In mind modeling we employ two different approaches for the steering vector calculation. The first and rather obvious one, used in simple mind, produces the 
steering vector as a weighted average of the individual ones. The second approach that takes into account priorities, called priority blending, is an enhanced version of the simple priority mind proposed in [21].

In simple mind, agent's velocity at each time frame is calculated as follows:

1. Compute steering vector $\boldsymbol{f}$ as a convex combination $\boldsymbol{f}=\sum w_{i} \boldsymbol{f}_{i}$, where $\boldsymbol{f}_{i}$, with $\left\|\boldsymbol{f}_{i}\right\|=1$, are the individual steering vectors from each simple behavior included in agent's mind. Weight values are generally agent- and time-dependent with weights corresponding to "prime" behaviors (i.e., those affecting collision avoidance: Obstacle Avoidance and Separation) being relatively higher than the remaining ones; see a detailed description in [24, $25]$.

2. New velocity is computed as:

$$
\boldsymbol{v}_{\text {new }}=c \cdot\left(\boldsymbol{v}_{\text {prev }}+\boldsymbol{f}\right), \text { where } c=\min \left\{\frac{v_{m}}{\left\|\boldsymbol{v}_{\text {prev }}+\boldsymbol{f}\right\|}, 1\right\},
$$

where, $v_{m}$ is the agent's maximum allowable velocity.

\section{Enhanced features of crowd modeling}

Crowd modeling, as described in [1] can be used to materialize a ship evacuation scenario adopting the advanced method of analysis proposed by IMO in circular $[26,2]$. Although this advanced method is more realistic than the simplified approach proposed in the same circulars, it is still subject to some restrictive assumptions and omissions as, e.g., ship motions, fire/smoke, crew assistance and passenger grouping effects which are collectively accounted via corrective safety factors. Aiming in the elimination of these restrictions, we herein enrich crowd modeling in VELOS with appropriate features, which are described in detail in the following sub-sections. These features include the introduction of new behaviors, as the Inclination behavior, modeling the effect of ship motions, the Enhanced Cohere behavior applied in passenger grouping, and the adoption of behavioral models and aids, such as the Triggers supporting crew assistance modeling. Finally, passenger's health index and ship's space availability are introduced for modeling smoke and/or fire influence on the evacuation process.

\subsection{Modeling Ship Motions \& Accelerations}

VELOS provides several interfaces for the consideration of ship motions and accelerations. Specifically, there are modules that allow importing of precomputed ship responses either in the frequency or time domain. Furthermore, there is also functionality for importing time histories of linear velocities and accelerations for selected points aboard a ship that are recorded with the aid of accelerometers. Thus, ship accelerations can be either estimated via numerical differentiation of ship motions or acquired from the experimental measurements. Generally, ship motions comprise time histories of the displacements of a specific point $\boldsymbol{P}$ of ship 
(usually ship's center of flotation) as well as time histories of ship rotational motions (pitch, roll and yaw). Using numerical differentiation we can calculate linear velocity $\left(\boldsymbol{v}_{p}\right)$ and acceleration $\left(\dot{\boldsymbol{v}}_{p}\right)$ of point $\boldsymbol{P}$ and angular velocity $\left(\boldsymbol{\omega}_{B}\right)$ and acceleration $\left(\dot{\boldsymbol{\omega}}_{B}\right)$ of the ship. Then, using the following well-known relations from rigid-body kinematics we can calculate velocity and acceleration at every point $\boldsymbol{Q}$ on ship: $\boldsymbol{q}=\boldsymbol{p}+\boldsymbol{\omega}_{B} \times \boldsymbol{r}_{p q}, \dot{\boldsymbol{v}}_{q}=\dot{\boldsymbol{v}}_{p}+\boldsymbol{\omega}_{B} \times\left(\boldsymbol{\omega}_{B} \times \boldsymbol{r}_{p q}\right)+\dot{\boldsymbol{\omega}}_{B} \times \boldsymbol{r}_{p q}$, where, $\boldsymbol{r}_{p q}$ is the vector formed by $\boldsymbol{P}$ and $\boldsymbol{Q}$.

The effects of ship motions on passengers and crew aboard are modeled in two ways as it is presented in detail in the sequel. The first simplified approach is based on a kinematic modeling that utilizes the ship motions while the second approach takes into account the dynamic nature of the phenomenon and relies on the availability of ship accelerations.

Inclination Behavior. Advanced evacuation analysis in VELOS is combining the availability of ship motion data with the so-called Inclination behavior that has been introduced, as a first layer, for considering the effect of ship motion on agent's movement. Precomputed ship-motion history is imported in VELOS through a suitable series of interfaces. Inclination behavior resembles in definition and effect the influence of a gravity field that would hinder agent motion accordingly. Specifically, we consider a static global force-vector $\boldsymbol{g}$ normal to deck's plane in the upright position of the ship. If the deck deviates from its upright position (i.e., non zero heel, and/or trim, angles), the projection of $\boldsymbol{g}$ on it will obviously acquire a non-zero value $\boldsymbol{g}_{p}$, which forms Inclination's steering vector as follows: $\boldsymbol{f}_{i}=\lambda(\phi) \frac{\boldsymbol{g}_{p}}{\left\|\boldsymbol{g}_{p}\right\|}$, where $\lambda(\phi)$ is an appropriate weight function depending on the angle $\phi$ formed between $\boldsymbol{g}$ and the normal to the deck plane. Inclination behavior is active when $\phi$ lies between two threshold angles: the lower threshold is used to discard plane motions with negligible effect on agent's motion, while values above the upper threshold lead to movement inability, as the limit of agent's balancing capabilities is surpassed. Threshold angles and the weight function $\lambda(\phi)$ are defined via experimental data; see, e.g., [27-29].

Motion Induced Interruptions (MII). During certain weather conditions, i.e., rough weather, walking and even more working in the ship becomes difficult and even the most experienced sailors will experience events where they must stop their activity, be it a specific task or merely standing, and take suitable measures to minimize the risk of injury, or more generally change their stance so that balance can be retained; these events are called, in pertinent literature, Motion-Induced Interruptions (MIIs). MIIs can be identified by considering the dynamic equations of motions of the person due to ship motion leading to the onset of loss-of-balance due to tipping or sliding. Baitis et al [30] and Graham et al $[31,32]$ have proposed the following relations for the consideration of tips to port or starboard. Specifically, a tip to port will occur if: $T_{L A T p}=\frac{1}{g}\left(\frac{1}{3} h \ddot{\eta}_{4}-\ddot{D}_{2}-g \eta_{4}-\frac{l}{h} \ddot{D}_{3}\right)>\frac{l}{h}$, and analogously for tip to starboard. Similarly, the following tipping coefficients can be derived when consider- 
ing tips to the aft or fore part of the ship: $T_{L O N a}=\frac{1}{g}\left(\ddot{D}_{1}+\frac{1}{3} h \ddot{\eta}_{5}-\frac{d}{h} \ddot{D}_{3}\right)>\frac{d}{h}$ and analogously for tip to fore.

In the above equations, $\eta_{1}$ (surge), $\eta_{2}$ (sway), and $\eta_{3}$ (heave) stand for the translational while $\eta_{4}$ (roll), $\eta_{5}$ (pitch) and $\eta_{6}$ (yaw) stand for the rotational components of ship motion along the $x-, y-$ and $z$ - axis of the shipcoordinate system, respectively, see Fig. 3. Furthermore, $\boldsymbol{D}=\left(D_{1}, D_{2}, D_{3}\right)=$ $\left(\eta_{1}, \eta_{2}, \eta_{3}\right)+\left(\eta_{4}, \eta_{5}, \eta_{6}\right) \times(x, y, z)$ denotes the displacement of point $\boldsymbol{P}(x, y, z)$. Finally, symbols $l, h$ and $d$ denote the half-stance length, the vertical distance to person's center of gravity and half-shoe width respectively as shown in Fig. 2. Typical values for $\frac{l}{h}$ lie in the interval $(0.20,0.25)$ while for $\frac{d}{h}$ lie in $(0.15,0.17)$.
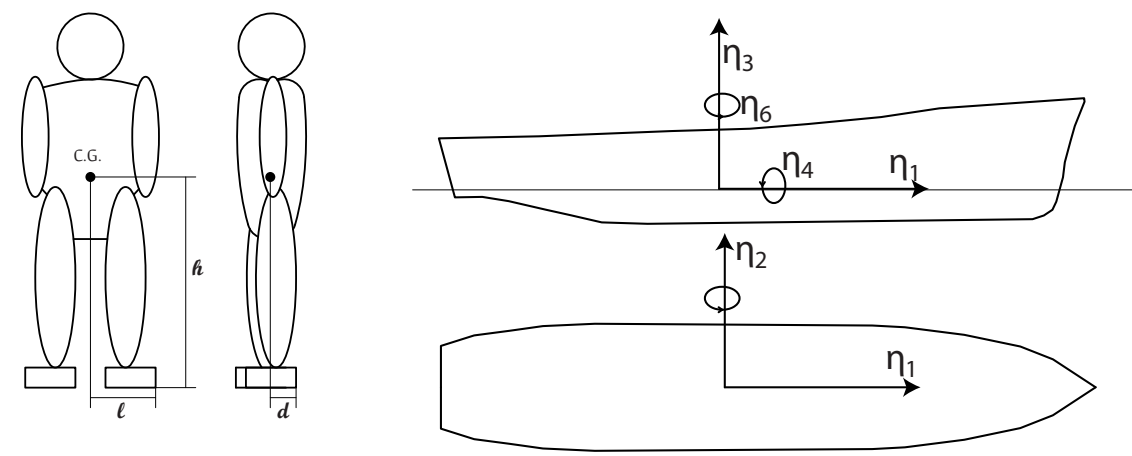

Fig. 2. Person C.G., half-stance and half-shoe width

Fig. 3. Ship coordinate system

Taking into account the above discussion concerning tipping coefficients, the effect of ship motions on passenger movement is implemented in the following way:

1. Adjustment $\tilde{v}_{m}$ of the maximum allowable velocity $v_{m}$ according to the following rule: $\tilde{v}_{m}=k \cdot v_{m}$, where

$k= \begin{cases}1, & \text { if } T_{L A T}<0.20 \wedge T_{L O N}<0.15 \\ \left(-20 T_{L A T}+5\right), & \text { if } 0.20<T_{L A T}<0.25 \wedge T_{L O N}<0.15 \\ \left(-20 T_{L A T}+5\right)\left(-50 T_{L O N}+8.5\right), & \text { if } 0.20<T_{L A T}<0.25 \wedge 0.15<T_{L O N}<0.17 \\ \left(-50 T_{L O N}+8.5\right), & \text { if } T_{L A T}<0.20 \wedge 0.15<T_{L O N}<0.17 \\ 0, & \text { if } T_{L A T}>0.25 \wedge T_{L O N}>0.17\end{cases}$

The values of $k$ are depicted graphically in Figure 4 .

2. Adjustment of $w_{i}$ weight values in computation of the steering vector. A typical scenario would include a $10 \%$ increase of the wander behavior contribution and a corresponding decrease in Obstacle Avoidance and Separation contribution.

3. Adjustment of the parameters of each individual steering behavior. 


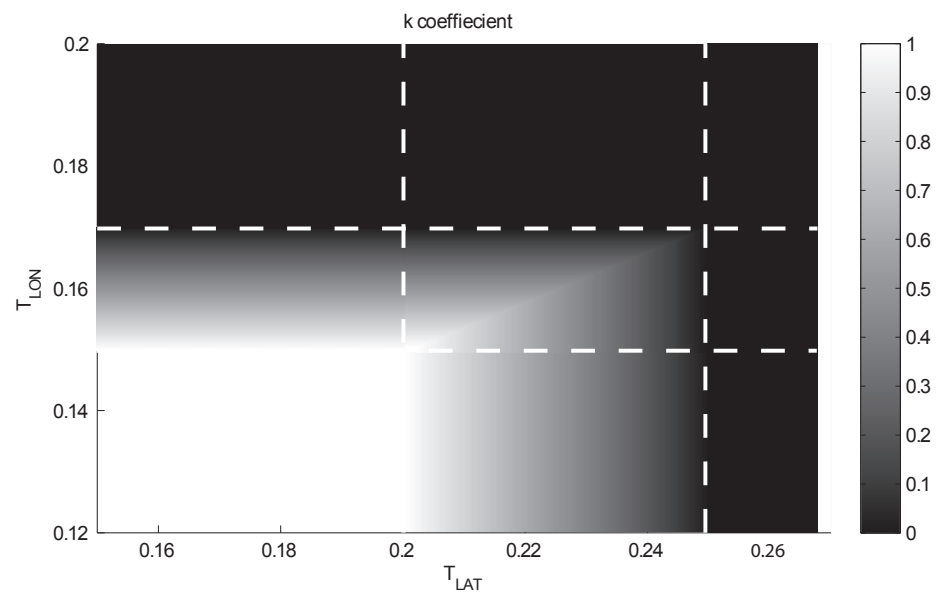

Fig. 4. Colorplot of k-coefficient values

\subsection{Passenger grouping}

Passenger grouping in VELOS, as presented in [24], is based on the EnhancedCohere behavior which constitutes an enhancement of the standard Cohere behavior. Enhanced-Cohere behavior is responsible for keeping together agents that are not only geometrically close to each other (as in the standard Cohere behavior), but also belong to the same group, e.g., a family, a crew guided group, etc. For this purpose, each agent is endowed with an ID in the form of a common length binary representation and the new velocity vector of every agent is obtained by applying the standard Cohere calculations on the subset of the neighboring agents that belong to the same group. Our implementation of standard Cohere behavior, assuming an agent's position at point $\mathbf{p}$ and the remaining group members locations at $\mathbf{p}_{i}$, respectively, produces a steering vector along the direction of $\mathbf{s}-\mathbf{p}$ where $\mathbf{s}$ is calculated as:

$$
\mathbf{s}=\frac{1}{\sum_{i} w_{i}} \sum_{i} w_{i} \mathbf{p}_{i}, \text { where } w_{i}=\frac{1}{\left\|\mathbf{p}-\mathbf{p}_{i}\right\|}
$$

In this way, by blending properly the Cohere behavior we can produce different grouping levels which can be categorized as follows:

Grouping Level 0: In this level, grouping is formed indirectly, via a common short-term target for the group members, as, e.g., followers of the same leader, or through the usage of the standard Cohere behavior.

Grouping Level 1: The members of the group are endowed with an ID and the Enhanced-Cohere behavior described above. Group cohesion is maintained only among nearby agents (within Cohere's neighborhood) sharing a common ID. However, if a member of the group gets out of the Cohere behavior's neighborhood, the remaining members will take no action. 
Grouping Level 2: The members of the group are endowed with the same properties as in Level 1 and moreover at least one member (e.g., the group leader) has the responsibility of checking group's integrity. In this way, cohesion of the group is maintained, since if a member of the group is lost the responsible agent will take some corrective action, as to wait for the lost member to join the group or to search for finding the lost member.

\subsection{Crew assistance}

Crew-Assistance behavior [24] is materialized by affecting the simple- or prioritymind mechanism in two ways, either by using Triggers or via the Guide Operation.

A Trigger attached to a crew agent is a scene object and at the same time a scene area (Trigger Neighborhood or TN) that, when visited by a passenger agent, a prescribed list of actions or property changes, the so called Trigger Actions or TAs, are applied to the agent. A TA example could be the following: if passenger density at the chosen TN exceeds a prescribed limit, the TA enables the crew agent to redirect passengers towards the closest muster station along a path different from the main escape route; see scenario 3 in $\S 4.1$.

Guide Operation is materialized through the Enhanced-Cohere behavior and the basic Leader-Follow behavior. A Guide-Operation example could involve a crew member that is ordered by the officer in charge to guide a group of passengers from a specific site to the closest muster station along a path different from that provided by the evacuation plan; see see scenario 2 in $\S 4.1$.

Furthermore improvement of Crew Assistance services could be provided by properly combining Triggers with Guide Operation. An example of this combined operation could involve a crew member that is charged to guide a group of passengers blocked at a space where a fire event is evolved.

\subsection{Influence of smoke, heat and toxic fire products}

VELOS offers the possibility to model a fire event during evacuation process by permitting passengers/crew to be influenced by smoke, heat and toxic fire products that are present in fire effluent. This is achieved by:

- importing precomputed time-series of fire products, according to different methods for calculating fire growth and smoke spread in multiple compartments; see, e.g., [33, 34],

- setting the time of fire or explosion (before, simultaneously or after the evacuation starting time),

- modeling the influence of fire products on the behavioral model of agents with the aid of the Function Health_ Index presented below,

- visualizing the fire products in the synthetic world.

Function Health_Index: In order to model the influence of fire products on agents we introduce the Health Reduction Rate function as follows:

$$
H R R(t)=F\left(a T(t)+b C_{C O}(t)\right), \quad(\text { Health_units } / \text { sec })
$$


where, $F$ describes the used functional model, $T$ is the temperature $\left({ }^{\circ} C\right)$ and $C_{C O}$ the carbon monoxide concentration (ppm) of the space where the agent is at the time $\mathrm{t}$ (see §4.2). We introduce now the Health Index function as follows

$$
H I(t)=1-\int_{0}^{t} F\left(a T(t)+b C_{C O}(t)\right) d t
$$

where, we have assumed that the initial Health Index of all agents is 1 . When the Health Index of an agent becomes zero the agent is considered dead. Moreover, when the Health Index of an agent deteriorates this also affects, by a suitable law, its maximum speed (ability of walking).

Function Space_Availability: In a typical ship evacuation simulation, the pathfinding module of VELOS computes the required path for each passenger to reach their designated muster station from their initial position. The employed algorithm is Dijsktra's shortest path algorithm [35] and is applied on ships topological graph where nodes correspond to ship spaces and edges to doors and/or passageways. Edge weighting between two connected nodes, in the simplest case, corresponds to the walking-distance between the two spaces' center points while this weighting scheme becomes more complex when space availability is considered. Specifically, ship spaces availability is connected and contribute to the edges' weighting implemented on the topology graph of ship spaces. For example, an increase of ambient temperature or CO concentration, or a visibility decrease in a certain space results in an increase of the weighting factors of the edges connected to the graph node representing this space. Consequently, paths passing through this particular space are less possible to be chosen by the path planning algorithm. Furthermore, when going beyond certain temperature, $\mathrm{CO}$ concentration and visibility thresholds, the corresponding space(s) is(are) rendered unavailable, i.e. removed from the topological graph.

\section{Test cases}

In this section we use VELOS for performing evacuation analysis for a RO-RO passenger ship:

- with and without crew assistance and grouping behaviors, and

- with and without a concurrent fire event.

Furthermore, we also examine the effect of ship motions on passengers' movement in the test case described in $\S 4.3$.

\subsection{Crew Assistance \& Grouping}

In the first test case examined, one hundred passengers are located in the cabins of Deck 5 (see Fig. 5) of the aft. vertical zone of a ship, while Muster Station is located on Deck 7. Population demographics are as proposed in [2]. For every simulation run we distribute randomly the population in the aforementioned 
areas. Three variations of the above scenario are simulated 3000 times each. For each variation, we compute the travel time required for all passengers to reach Muster Station as well as cumulative arrival time corresponding to the percentage of passengers reaching Muster Station for each time unit.

In the first variation (Scenario 1), passengers follow the designated escape route without crew assistance; Fig. 6 provides a snapshot of the evacuation process. The other two variations involve crew assistance. In Scenario 2 passengers are directed by two crew members to follow two distinct routes (see Fig 7), while in Scenario 3 a crew member monitors passengers' density at a specified place and, whenever congestion is likely to arise, he/she redirects a group of passengers towards a secondary escape route; see Fig. 8. In both cases, crew assistance is materialized through Triggers, which in Scenario 2 involves TAs applied to all passengers passing through the corresponding TN, while in Scenario 3 TAs are of dynamic character as a result of the attached density sensor.

Figure 9 depicts the average of the cumulative arrival time for each scenario. As it can easily be seen from this figure, Scenarios 2 and 3, based on crewassistance \& grouping, achieve a considerably better performance compared to Scenario 1. Among Scenarios 2 and 3, the latter is marginally better as a result of the dynamic crew-assistance policy adopted. Analogous conclusions can be drawn from Fig. 10, where the distributions of travel-time of the three scenarios are depicted. Average travel time for Scenarios 1, 2 and 3 are equal to $147 \mathrm{~s}$, $112 \mathrm{~s}$ and $113 \mathrm{~s}$, respectively. Moreover, in Scenarios 2 and 3 travel-time distribution is narrow-banded, which reflects the effectiveness of the adopted evacuation processes versus that of Scenario 1.
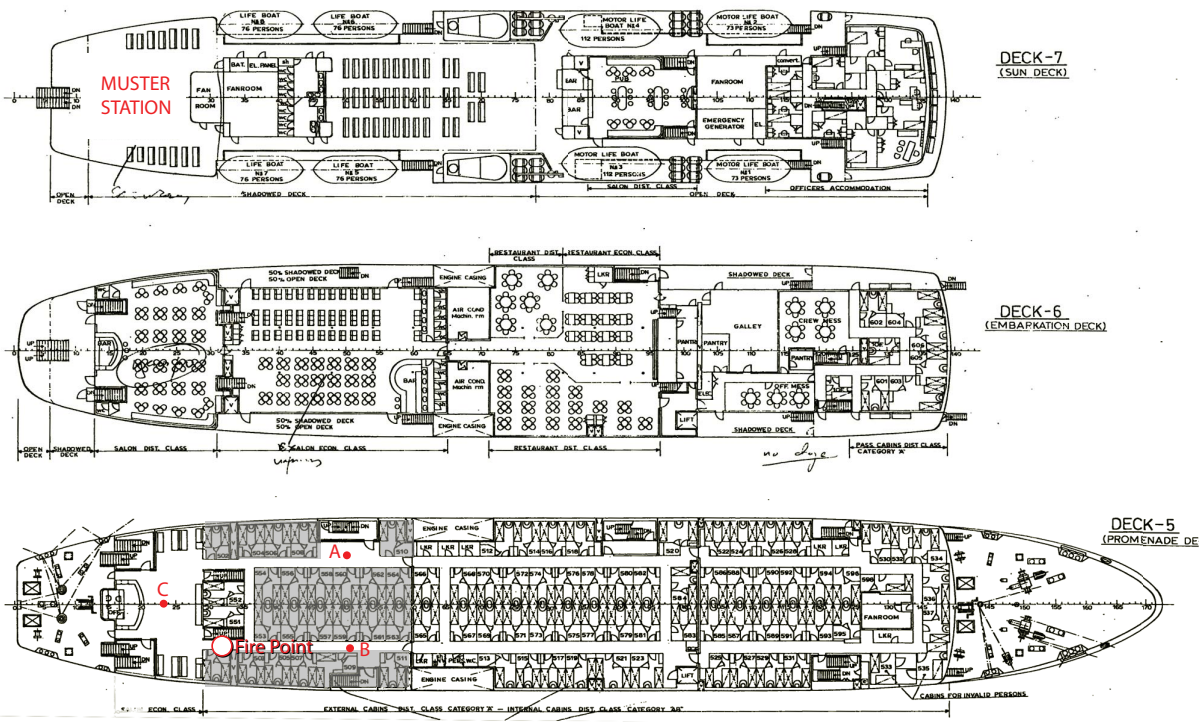

Fig. 5. General Arrangement and passengers distribution at the aft. vertical zone 


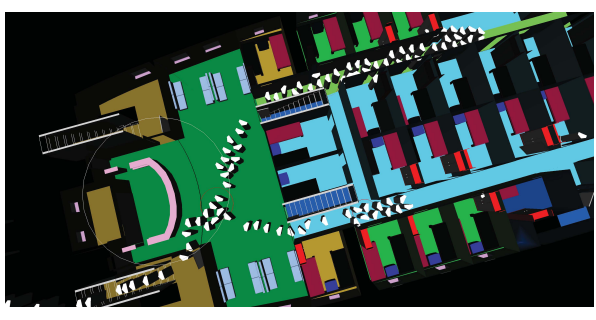

Fig. 6. Scenario 1

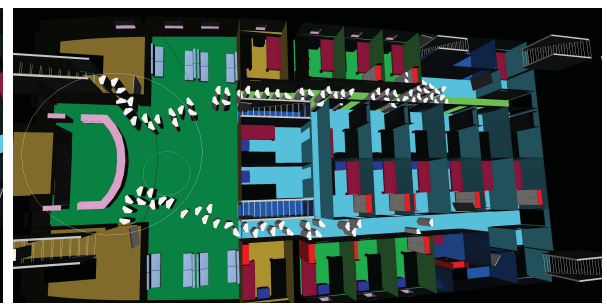

Fig. 7. Scenario 2

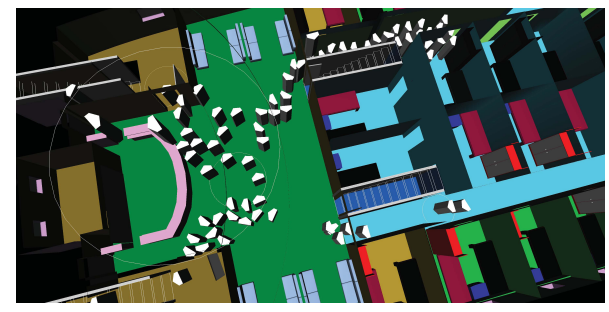

Fig. 8. Scenario 3

\subsection{Fire Event}

In this test case, we have the same arrangement and passenger distribution with the first test case; see Fig. 5. Population demographics are as proposed in [2]. A fire event occurs simultaneously with the beginning of the evacuation process. The initial fire site is located on deck 5 and depicted in Fig. 5. The fire propagation, along with temperature distribution, Carbon Monoxide (CO) concentration and visibility due to smoke has been precomputed [34] for all affected spaces on deck 5 and the time history of all corresponding quantities has been imported to VELOS. Fire and its products (temperature, CO concentration and visibility-degradation due to smoke) affect both the availability of ship spaces and the movement capabilities of passengers and their health. Space availability changes are implemented via the edge weighting mechanism described in $\S 3.4$

For every simulation run we distribute randomly the population in the aforementioned areas. The fire scenario under consideration is simulated 360 times and for each run, we record the travel time required for all passengers to reach Muster Station and compute the cumulative arrival time corresponding to the percentage of passengers reaching Muster Station for each time unit. As illustrated in Fig. 11 the passengers reaching muster station are around $30 \%$ less when compared to the evacuation without the fire event. This is caused by the fire-blockage of passage ways and the resulting fatalities. Furthermore, the slight acceleration of the evacuation process depicted in the same figure for the fireevent example case is due to the fact that the effective evacuation population 

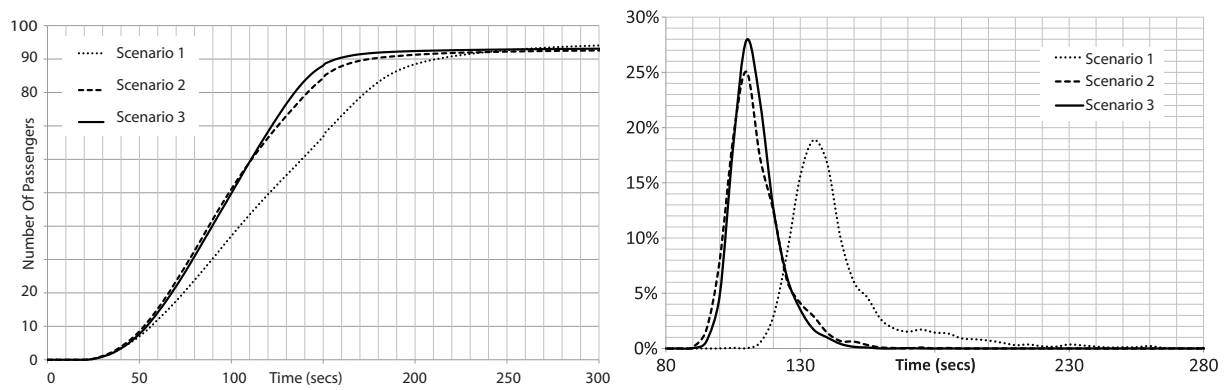

Fig. 9. Average cumulative arrival time for Fig. 10. Travel time distribution for SceScenarios 1, 2 and 3 narios 1,2 and 3

has been reduced due to the effects of the fire incident and thus the available spaces and pathways are used by less evacuating passengers.

\subsection{Ship Motions' Effect}

This last test case examines passengers' movement on Deck 5 of the same RO-RO passenger ship with and without ship motions' effect consideration. Specifically, we simulate the movement of two groups of passengers (20 persons) from points $\mathrm{A}$ and $\mathrm{B}$ respectively, to point $\mathrm{C}$ (see Fig. 5) in still water, and at a sea state described by a wave spectrum with $4 \mathrm{~m}$ significant wave height, 11 sec. peak period and $90^{\circ}$ ship heading (beam seas). Ship responses were pre-computed and imported into VELOS using the SWAN seakeeping software package. The cases examined have as follows:

- Still water (No Waves),

- (Sea state as described above): Kinematic modeling of motion effects through inclination behavior,

- (Same sea state): Dynamic modeling using tipping coefficients implementation.

Figure 12 depicts the average cumulative arrival time to point $\mathrm{C}$ for each of the three example cases. Each of the test cases has been simulated 500 times and the average travel times and arrival rates at point $\mathrm{C}$ have been collected. As it can easily be seen from this figure the time required for the prescribed passengers movement is the least when we are in still water. The effect of the wavy sea state, which induces ship motions and hinders passengers movement is illustrated with the right-shifting of the remaining two curves. The total travel time needed for both inclination behavior and tipping coefficient modeling is about the same $(\approx 70$ secs $)$ and considerably higher than the still water case $(\approx 50$ secs $)$, where, obviously, no motion effect is considered. However the arrival rate (slope) for the tipping coefficient modeling is steeper than the slope of the curve corresponding to the kinematic approach. 


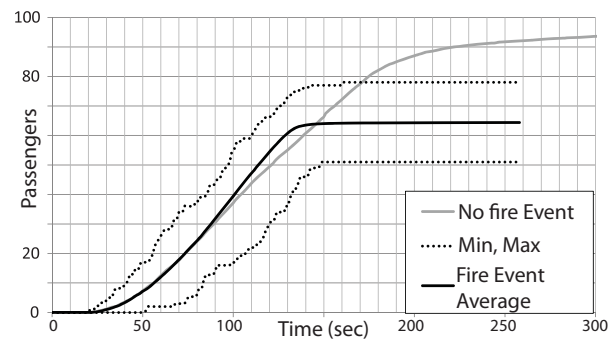

Fig. 11. Ship evacuation with and without a fire event

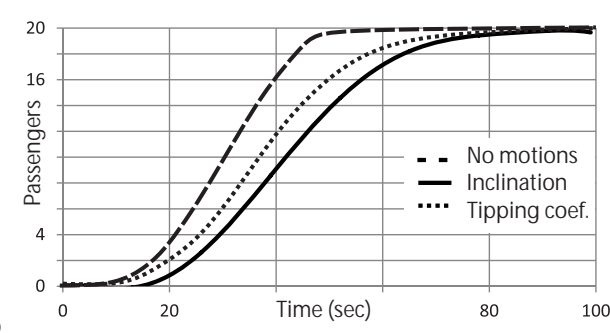

Fig. 12. Average cumulative arrival time for test cases 1, 2 and 3

\section{Current Work - Dynamic Grouping}

As the assumption that passengers have knowledge of the full route to muster stations is not realistic, we aim in further developing the grouping behaviors and guiding functionality within groups so that a more realistic path-finding can be accomplished. Towards this goal, we briefly present here the current development of Dynamic Grouping.

Dynamic Grouping extends the Enhanced-Cohere behavior described in $\S 3.2$. Additionally to group IDs, dynamic grouping uses the notion of a group leader which, obviously, shares the same ID with the corresponding group and possesses the leader tag. The steering vector, in this case, is a weighted average of the steering vector in Eq. 3 and the vector $\frac{\mathbf{l}-\mathbf{p}}{\|\mathbf{l}-\mathbf{p}\|}$, where $\mathbf{l}, \mathbf{p}$ correspond to leader's and agent's positions respectively.

Dynamic grouping permits changes in group membership when the group leader is not "visible" to a group member. When a member stops seeing the leader, it loses its ID and thus, stops belonging to a specific group. After that, the "lost" individual scans within its neighborhood for other leaders, i.e., agents look in their "view area" for other agents and choose to follow the leader that most of them are following. If one is found, the individual acquires the group ID of the leader closer to its position. If none is found, it switches its EnhancedCohere behavior to the standard one as described in $\S 3.2$.

The following subsection demonstrates the current development of the Dynamic Grouping behavior for the case of two groups with leaders in a simple space arrangement. "Visibility" in this generic example is implemented as a circular disc with a prescribed radius centered at the agent's position.

\subsection{Example Scenario}

The test environment comprises 4 consecutive spaces $(7.5 \times 10 \mathrm{~m}$ each $)$ and two initial groups. The first group (black group) is initially positioned at space A while the second group (gray group) resides in space B as shown in Fig. 13. Black group comprises, excluding its leader (colored white with black outline), 18 members and the gray group comprises 13 members. The target space for both 
groups is space D and only group leaders have knowledge of the required path. All group members are endowed with the following set of steering behaviors:

\section{Obstacle Avoidance,}

2. Separation,

3. Wander, and

4. Dynamic Cohere

Both group leaders are equipped with the above set of behaviors with the addition of the Path-Following steering behavior.

We demonstrate the dynamic grouping in a simple generic example for 5 test cases with different group leaders' velocities and/or initial positions along their predefined path towards the target space.

The first 3 test cases investigate the effect of leaders speed. In Figs. 1416 group members have maximum allowable speeds according to the statistical distributions prescribed in [2] while the black group leader has a speed equal to the black group members' average speed. In Fig. 14 the gray group leader has a maximum speed $(1.2 \mathrm{~m} / \mathrm{s})$ lower than the average of its corresponding group. As a consequence its group members retain their membership (with the exception of a single slow individual) through the whole experiment. In Fig. 15 the gray group leader has a maximum speed $(1.4 \mathrm{~m} / \mathrm{s})$ equal to the average of its corresponding group. In this case the slower members lose their group membership as their leader leaves their visibility area. Finally, in Fig. 16, the gray group leader is considerably faster $(1.6 \mathrm{~m} / \mathrm{s})$ than the average speed leading to a significant reduction of its group size at route's halfway.

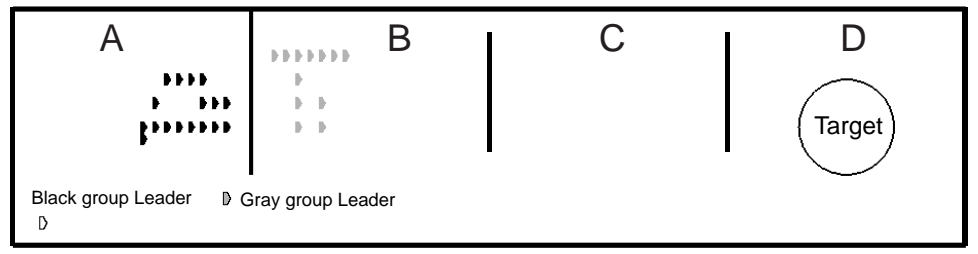

Fig. 13. Generic example: spaces and groups' arrangement

The second set of test cases comprises two experiments: One with leaders having initial position closer to their corresponding group while for the second test we have shifted the black group leader's position on the path towards the target space (see Fig. 18). In this experiment both leaders' speed is equal to their groups' average speed. In the first test case (Fig. 17), as expected, all members retain their group membership while in the second one both groups merge forming one group behind the gray group leader. There is an exception of a black individual that manages not to lose sight of its initial leader (see Fig. 18). 


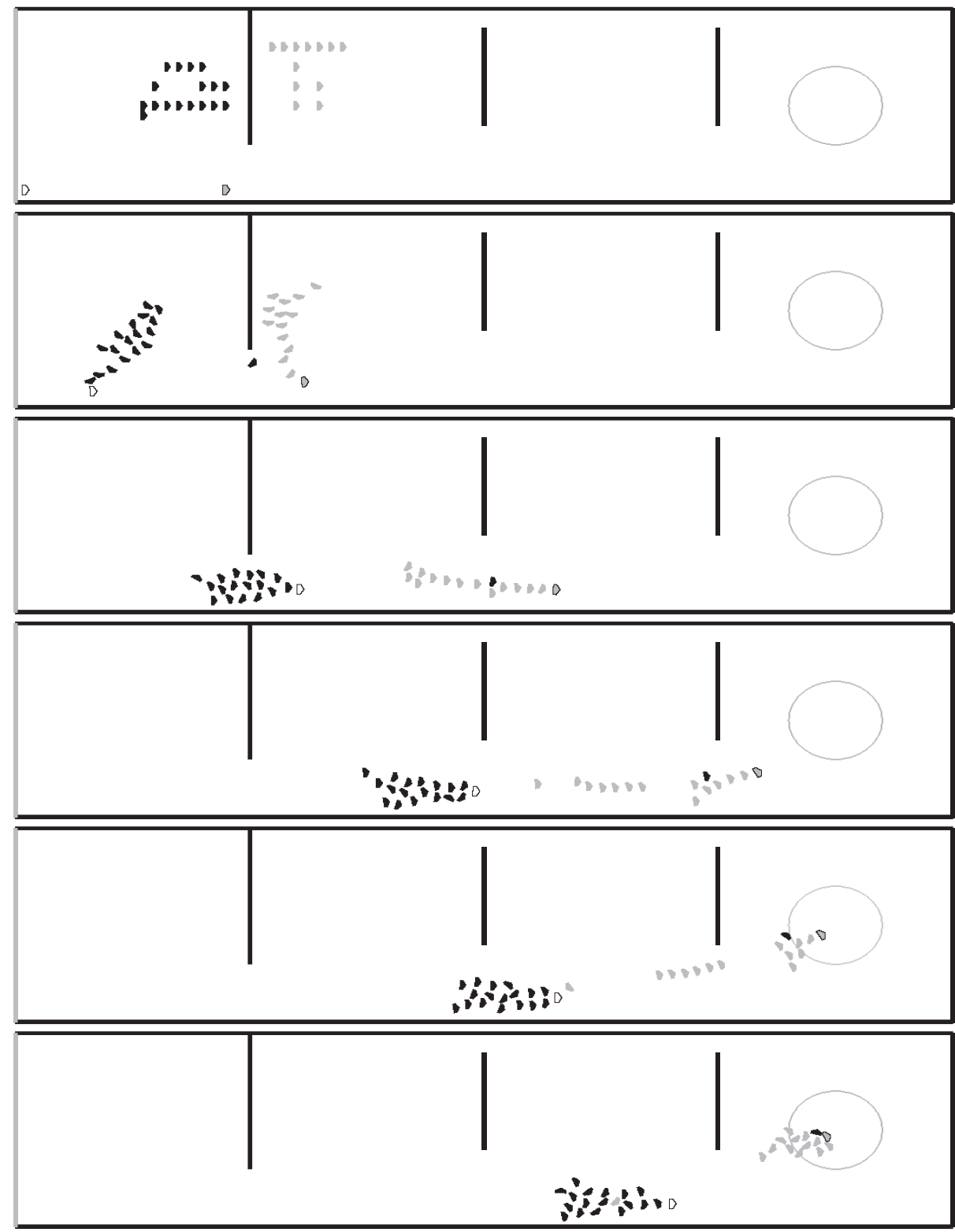

Fig. 14. Gray Group Leader: low speed, $1.2 \mathrm{~m} / \mathrm{s}$ 


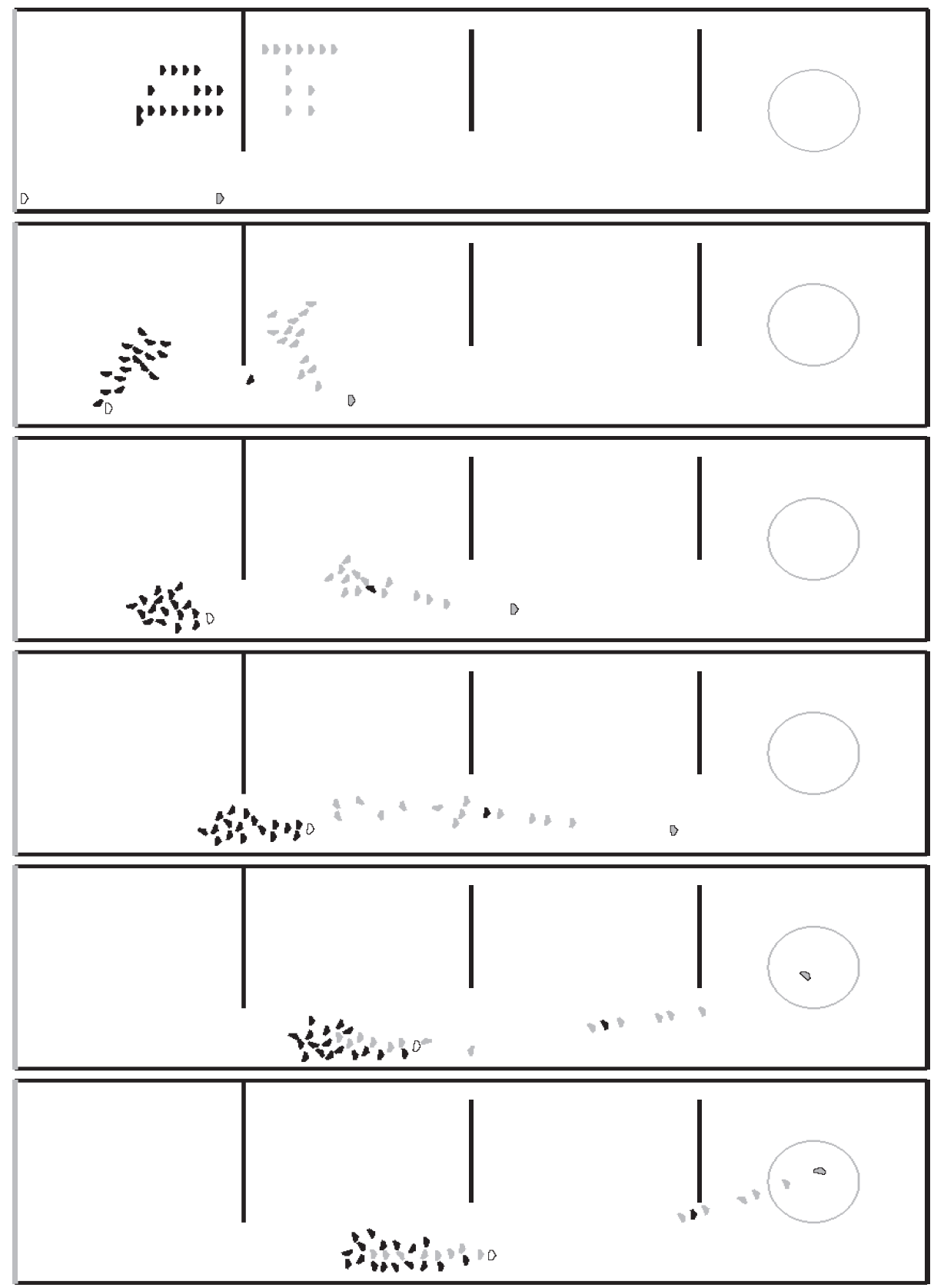

Fig. 15. Gray Group Leader: medium speed, $1.4 \mathrm{~m} / \mathrm{s}$ 


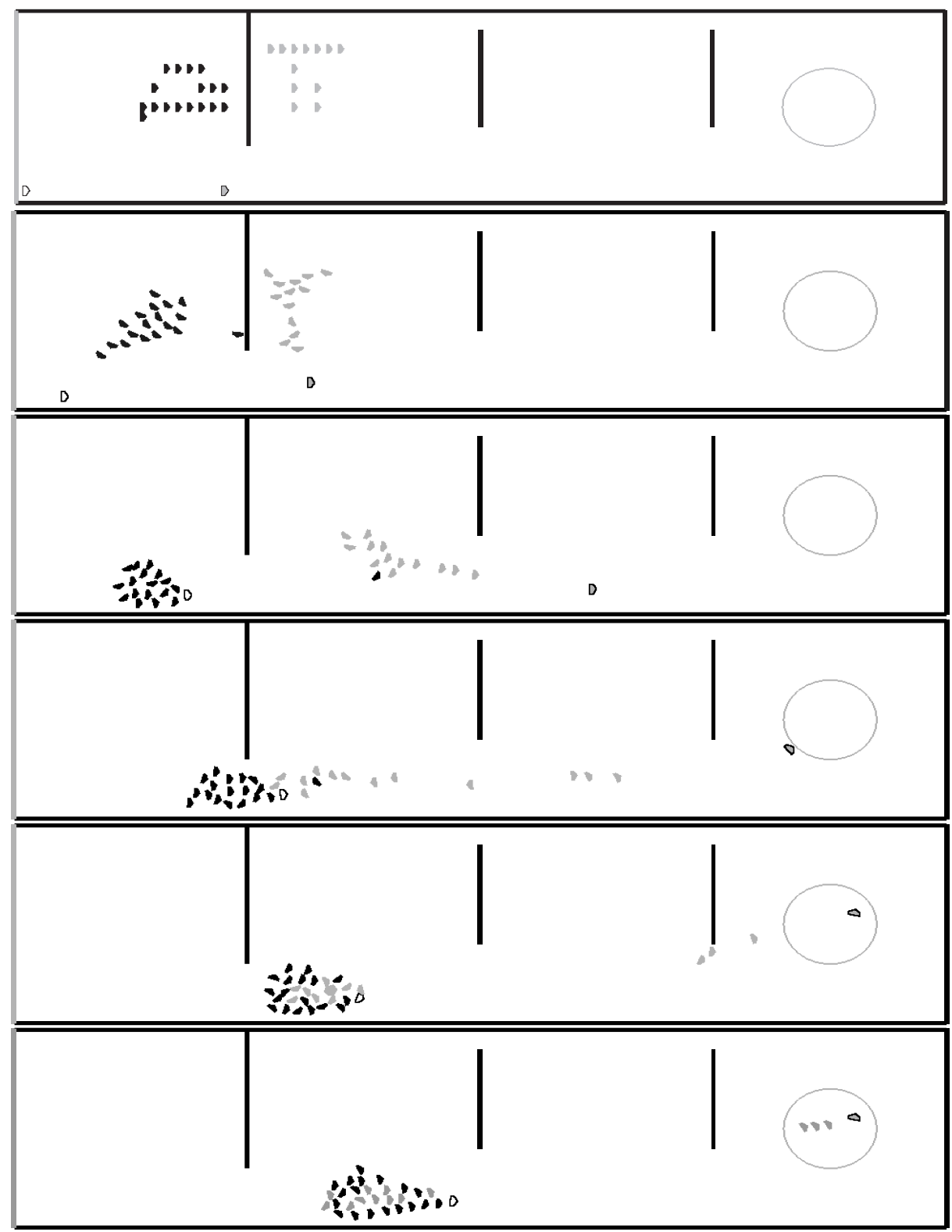

Fig. 16. Gray Group Leader: high speed, $1.6 \mathrm{~m} / \mathrm{s}$ 


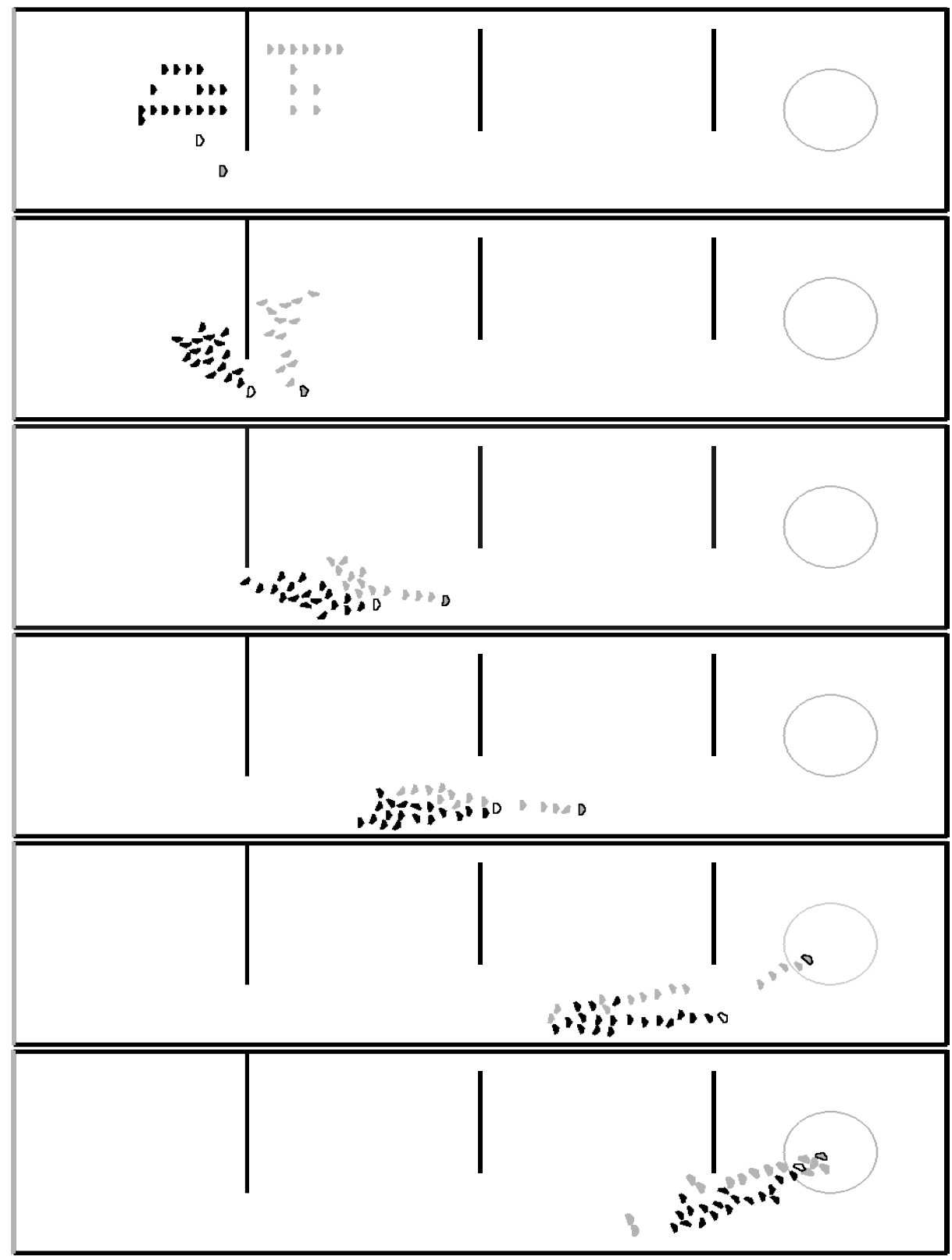

Fig. 17. Group Leaders: Close to their group 


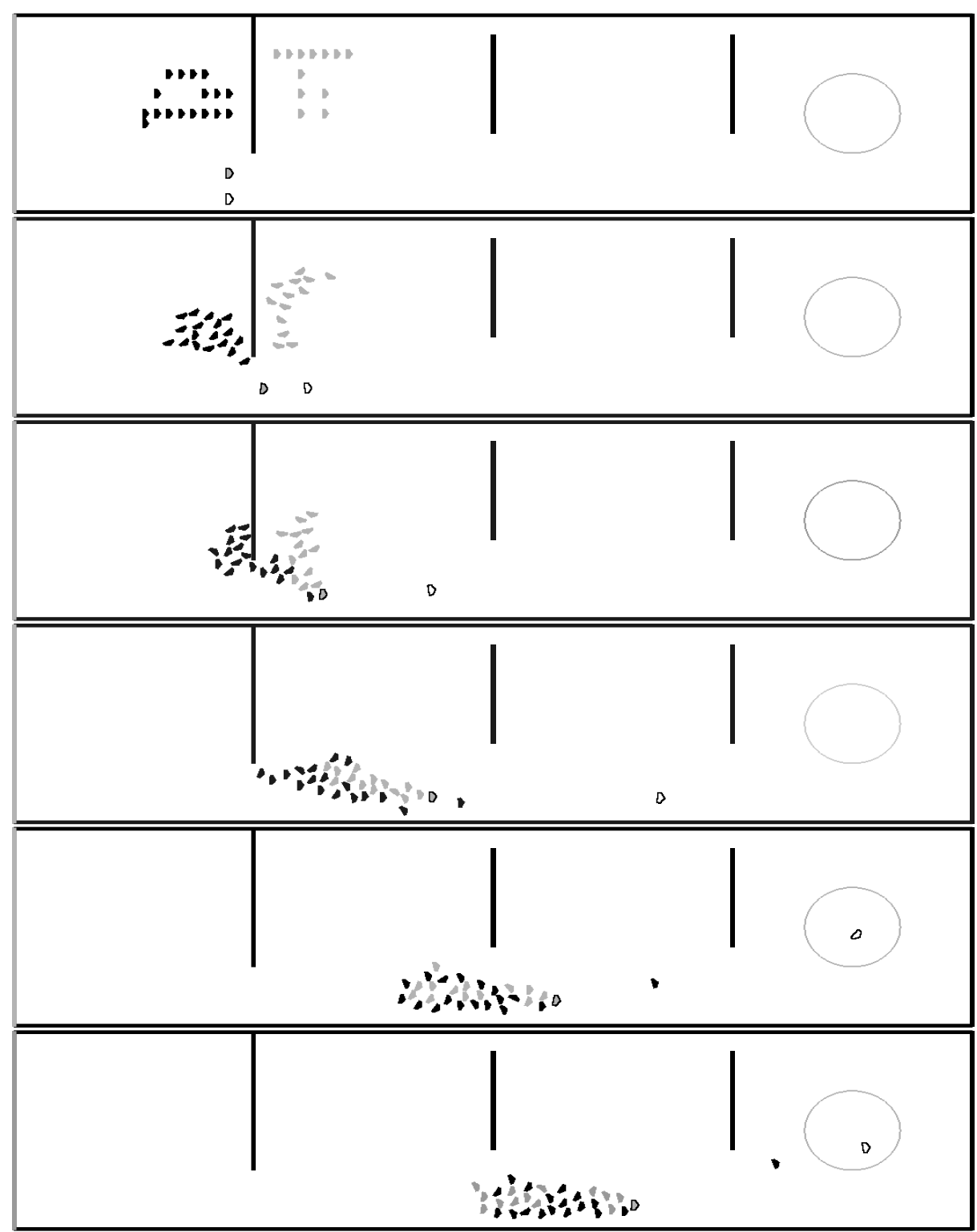

Fig. 18. Black Group Leader: Shifted $7.5 \mathrm{~m}$ towards the target space 


\section{Conclusion}

The present paper provides a description of the enhanced crowd modeling approach employed in VELOS for the performance of ship evacuation assessment and analysis based on the guidelines provided by IMO's Circular MSC $1238 / 2007$ [2]. Although the evacuation scenarios, proposed in [2] address issues related to the layout of the ship and passenger demographics, they do not touch issues arising in real emergency conditions, such as unavailability of escape arrangements (due to flooding or fire), crew assistance in the evacuation process, family-group behavior, ship motions, etc.

We have presented VELOS' components and functionalities including a brief description of the employed crowd modeling approach for the performance of ship evacuation assessment \& analysis. The VELOS novel features include: the modeling of ship motions \& accelerations, passenger grouping and crew assistance and the influence of smoke, heat and toxic fire products. The examples presented include ship evacuation test cases investigating the effects of crew assistance, passenger grouping and fire incidents. Furthermore, an additional test case demonstrating the effects of ship motions on passengers movement is also included. Finally, our ongoing work extending grouping behavior with dynamic characteristics has been also presented.

As a concluding remark, we would like to note that an obvious next step in the development process is the inclusion and assessment of dynamic grouping in a more realistic ship test case. This, however, requires further development of the "visibility" mechanism and elaboration of the factors that affect members' group-changing decision.

\section{Acknowledgments}

This research has been co-financed by the European Union (European Social Fund - ESF) and Greek national funds through the Operational Program "Education and Lifelong Learning" of the National Strategic Reference Framework (NSRF) - Research Funding Program: THALIS-UOA (MIS 375891).

\section{References}

1. Ginnis, A.A.I., Kostas, K.V., Politis, C.G., Kaklis, P.D.: VELOS: A VR platform for ship-evacuation analysis. CAD, special issue on Computer Aided Ship Design 42(11) (2010) 1045-1058

2. I.M.O.: Guidelines for evacuation analyses for new and existing passenger ships. Msc.1/circ. 1238 edn. (30 October 2007)

3. Vanem, E., Skjong, R.: Designing for safety in passenger ships utilizing advanced evacuation analyses - a risk based approach. Safety Science 44 (2006) 11-35

4. Lee, D., Kim, H., Park, J.H., Park, B.J.: The current status and future issues in human evacuation from ships. Safety Science 41(10) (2003) 861-876 
5. Kim, H., Park, J.H., Lee, D., soon Yang, Y.: Establishing the methodologies for human evacuation simulation in marine accidents. Computers \& Industrial Engineering 46(4) (2004) 725-740

6. Valanto, P.: Time-dependent survival probability of a damaged passenger ship ii - evacuation in seaway and capsizing. Technical Report 1661, Hamburg, HSVA (2006)

7. Galea, E., Lawrence, P., Gwynne, S., Sharp, G., Hurst, N., et al, Z.W.: Integrated fire and evacuation in maritime environments. In: Proceedings of the 2nd International Maritime Conference on Design for Safety, Sakai, Japan. (2004) 161-170

8. Park, J., Lee, D., Kim, H., Yang, Y.: Development of evacuation model for human maritime casualty. Ocean Engineering 31(0) (2004) 1537-1547

9. Vassalos, D., Kim, H., Christiansen, G., Majumder, J.: A mesoscopic model for passenger evacuation in a virtual ship-sea environment and performance-based evaluation. In: Proceedings of Conference on Pedestrian and Evacuation Dynamics, Duisburg. (2001)

10. Vassalos, D., Guarin, L., Vassalos, G., Bole, M., Kim, H., Majumder, J.: Advanced evacuation analysis - testing the ground on ships. In: Proceedings of Conference on Pedestrian and Evacuation Dynamics, Greenwich. (2003)

11. Drager, K., Orset, S.: Evac - the mustering and evacuation computer model resulting from the briteeuram project mepdesign. In: Proceedings of Conference on Pedestrian and Evacuation Dynamics, Duisburg. (2001) 355-368

12. Klupfel, H., Meyer-Konig, M., Wahle, J., Schreckenberg, M.: Microscopic simulation of evacuation processes on passenger ships. In: Theoretical and practical Issues on Cellular Automata. Springer (2000) 63-71

13. Thalmann, D., Musse, S.: Crowd Simulation. Springer (2007)

14. Helbing, D., Farkas, I., Viscek, T.: Simulating dynamical feaatures of escape panic. Nature 407 (2000) 487-490

15. Henderson, L.: The statistics of crowd fluids. Nature 229 (1971) 381-383

16. Helbing, D., Molnar, P., Farkas, I., Bolay, K.: Self-organizing pedestrian movement. Environment and Planning B: Planning and Design 28 (2001) 361-383

17. Hughes, R.: The flow of human crowds. Annual Review of Fluid Mechanics 224 (1970) 120-123

18. Gardner, M.: Mathematical games: Conway's game of life. Scientific American 407 (2000) 487-490

19. Blue, V., Adler, J.: Cellular automata microsimulation of bi-directional pedestrian flows. Transportation Research Board 1678 (2000) 135-141

20. Reynolds, C.: Flocks, herds and schools: A distributed behavioral model. Computer Graphics 21(4) (1987) 25-34

21. Reynolds, C.W.: Steering behaviors for autonomous characters. In: GDC'99 (Game Developers Conference). (1999)

22. Helbing, D., Molnár, P.: Social force model for pedestrian dynamics. Phys. Rev. E 51 (May 1995) 4282-4286

23. Green, R.: Steering behaviors. In: SIGGRAPH 2000 Conference Proceedings. (2000)

24. Kostas, K., Ginnis, A.I., Politis, C., Kaklis, P.: Use of VELOS platform for modelling and accessing crew assistance and passenger grouping in ship-evacuation analysis. In: Sustainable Maritime Transportation and Exploitation of Sea Resources. Eds E. Rizzuto, C. Guedes Soares. Volume 2. (2011) 729-736

25. Kostas, K.: Virtual Reality Kernel with Support for Ship Life-cycle Modeling. PhD thesis, Naval Architecture \& Marine Engineering, NTUA (2006) 
26. I.M.O.: Interim Guidelines for evacuation analyses for new and existing passenger ships, MSC/Circ. 1033. (June 2002)

27. Bles, W., Nooy, S., Boer, L.: Influence of ship listing and ship motion on walking speed. In: Proceedings of Conference on Pedestrian and Evacuation Dynamics. (2001)

28. Crossland, P.: The influence of ship motion induced lateral acceleration on walking speed. In: Proceedings of the 2nd International Conference on Pedestrian and Evacuation Dynamics, Greenwich. (2003)

29. Crossland, P., Evans, M.J., Grist, D., Lowten, M., Jones, H., Bridger, R.S.: Motioninduced interruptions aboard ship: Model development and application to ship design. Occupational Ergonomics 7(3) (2007) 183-199

30. Baitis, A.E., Holcombe, F.D., Conwell, S.L., Crossland, P., Colwell, J., Pattison, J.H.: 1991-1992 Motion Induced Interruptions (MII) and Motion Induced Fatigue (MIF) experiments at the Naval Biodynamics Laboratory. Technical Report CRDKNSWC-HD-1423-01, Bethesda, MD: Naval Surface Warfare Center, Carderock Division. (1995)

31. Graham, R.: Motion-induced interruptions as ship operability criteria. Journal of Naval Engineers 102(2) (1990) 65-71

32. Graham, R., Baitis, A.E., Meyers, W.: On the development of seakeeping criteria. Journal of Naval Engineers 104(3) (1992) 259-275

33. Rein, G., Barllan, A., Fernandez-Pell, C., Alvares, N.: A comparison of three models for the simulation of accidental fires. Fire Protection Engineering 1 (2006) 183-209

34. McGrattan, K., Klein, B., Hostika, S.: Fire Dynamics Simulator. NIST. (2007) Maryland: NIST Special Publication 1019-5.

35. Dijkstra, E.W.: A note on two problems in connexion with graphs. Numerische Mathematik 1 (1959) 269-271 